BRAZILLAN JOURNAL

www.bjournal.com.br
ISSN 0100-879X

Volume 43 (3) 182-267 March 2011

BIOMEDICAL SCIENCES

AND

CLINICAL INVESTIGATION

Braz J Med Biol Res, March 2011, Volume 44(3) 258-262

doi: 10.1590/S0100-879X2011007500013

\title{
Adherence to drug therapy in kidney disease
}

E.J.C. Magacho, L.C. Ribeiro, A. Chaoubah and M.G. Bastos

The Brazilian Journal of Medical and Biological Research is partially financed by

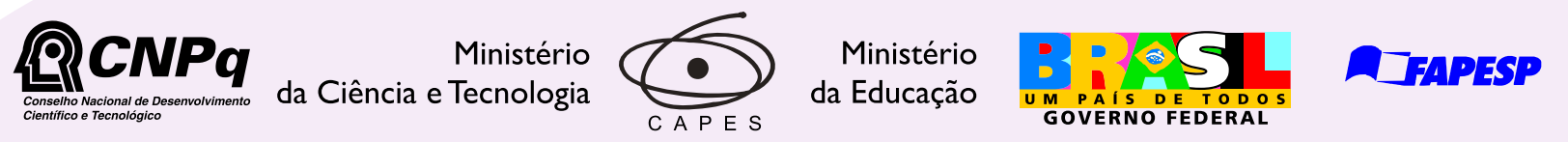

Institutional Sponsors

sup?o
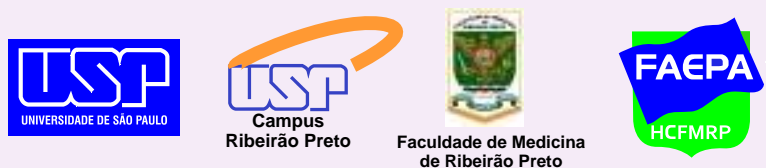

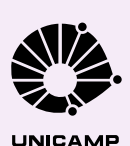

$\oplus$ SHIMADZU

GE Healthcare
Hotsite of proteomics metabolomics developped by:

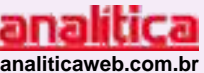

$\underset{\text { SCIENTIFIC }}{\text { Thermo }}$ 


\title{
Adherence to drug therapy in kidney disease
}

\author{
E.J.C. Magacho ${ }^{1}$, L.C. Ribeiro ${ }^{2}$, A. Chaoubah² and M.G. Bastos ${ }^{3}$ \\ ${ }^{1}$ Hospital Universitário, Universidade Federal de Juiz de Fora, Juiz de Fora, MG, Brasil \\ ${ }^{2}$ Departamento de Estatística, Instituto de Ciências Exatas, \\ Universidade Federal de Juiz de Fora, Juiz de Fora, MG, Brasil \\ ${ }^{3}$ Departamento de Nefrologia, Faculdade de Medicina, \\ Universidade Federal de Juiz de Fora, Juiz de Fora, MG, Brasil
}

\begin{abstract}
Non-adherence to drug therapy has not been extensively studied in patients with chronic kidney disease (CKD). The objective of the present study was to identify determinants of non-adherence to drug therapy in patients with CKD, not on dialysis. A prospective cohort study involving 149 patients was conducted over a period of 12 months. Adherence to drug therapy was evaluated by the self-report method at baseline and at 12 months. Patients who knew the type of drug(s) and the respective number of prescribed pills in use at the visit preceding the interview were considered to be adherent. Patients with cognitive decline were assessed by interviewing their caregivers. Mean patient age was $51 \pm 16.7$ years. Male patients predominated $(60.4 \%)$. Univariate analysis performed at baseline showed that non-adherence was associated with older age, more pills taken per day, worse renal function, presence of coronary artery disease, and reliance on caregivers for the administration of their medications. In multivariate analysis, the factors that were significantly associated with non-adherence were daily use of more than 5 pills and drug administration by a caregiver. Longitudinal evaluation showed an increase in non-adherence over time. Medication non-adherence was lower (17.4\%) at the baseline period of the study than after 1 year of the study $(26.8 \%)$. Compared to the baseline period, the percentage of adherent patients who became non-adherent (22\%) was lower than the percentage of non-adherent patients who became adherent (50\%). In CKD patients not on dialysis, non-adherence was significantly associated with the number of pills taken per day and drug administration by third parties. Adherence is more frequent than non-adherence over time.
\end{abstract}

Key words: Drug adherence; Chronic kidney disease; Drug therapy; Self-report; Nursing

\section{Introduction}

Chronic kidney disease (CKD) is a major public health problem and its diagnosis is greatly facilitated by the criteria of the Kidney Disease Outcomes Quality Initiative (K/DOQI) of the US National Kidney Foundation (1). According to the clinical practice guidelines of K/DOQI, CKD is defined for individuals with glomerular filtration rate $<60 \mathrm{~mL} \cdot \mathrm{min}^{-1} \cdot 1.73$ $\left(\mathrm{m}^{2}\right)^{-1}$, estimated at two different times with an interval of at least three months.

In Brazil, there are no population studies on the prevalence of CKD, but the causes and risk factors of CKD in our country are similar to those found in the United States, which leads us to believe that the disease also has a high prevalence in our country (2).

Slowing the rate of disease progression in the early stages of CKD is a major goal, together with monitoring and correcting its complications and comorbidities, and treating the underlying disease. However, to achieve these goals, adherence to treatment plays a major role, whereas non-adherence to prescribed drugs significantly increases morbidity and mortality in developing countries $(3,4)$.

According to the World Health Organization (WHO), "adherence is a person's behavior concerning taking medication, following a diet, and making changes in lifestyle in accordance with a medical or non-medical health professional recommendations" (5).

Non-adherence is considered to be a multidimensional phenomenon related to multiple factors, and its occurrence can adversely impact the course of the disease, particularly in cases of chronic disease (5). These factors are determined by the characteristics of each disease, patient's healthcare system, socioeconomic factors, treatment characteristics, and the health team who assists the patient; thus, considering patients as solely responsible for following treatment is misleading (6), mainly because some factors are inde-

Correspondence: E.J.C. Magacho, Hospital Universitário, Universidade Federal de Juiz de Fora, Rua José Lourenço Kelmer, 1300 , Salas 204 a 222, 36036-330 Juiz de Fora, MG, Brasil. E-mail: edsonmagacho@acessa.com

Received July 6, 2010. Accepted January 24, 2011. Available online February 4, 2011. Published March 7, 2011. 
pendent of their will, particularly the occurrence of different levels of cognitive decline (7-10), treatment of asymptomatic diseases, medication side effects, no clarification about the disease, and medication cost (11).

The methods used to assess adherence to drug therapy can be classified into direct and indirect. The direct method is based on analytical techniques that confirm if a given medication was taken at the dose and frequency prescribed by the identification of drug metabolites or chemical markers in the body (12). Indirect methods are those based on self-report, pill count, tablet refills, electronic monitoring (13), and Morisk's method, among others $(14,15)$.

So far, most of the studies on adherence in patients with kidney diseases have assessed non-adherence to immunosuppressive drugs as the cause of graft loss in transplanted patients, whereas few studies have been conducted on patients with CKD not yet on dialysis. The aim of the present study was to evaluate adherence to drug therapy and associated factors of non-adherence in patients with CKD, not on dialysis.

\section{Material and Methods}

In the present study, a cohort of patients who attended the outpatient service of Nephrology at Hospital Universitário, Universidade Federal de Juiz de Fora (HU/UFJF), was followed for 1 year from October 2007 to September 2008.
Patient care was provided in the setting of a secondary care university interdisciplinary clinic, with ongoing involvement of a nurse, a nephrologist, social workers, a psychologist, and nutritionists, all of them seeing the patients on the same day.

The inclusion criterion was age above 18 years. Patients older than 60 years underwent the Minimental State Examination (MMSE) and, when they were found to have impaired cognitive function test, the caregiver was invited to answer the questionnaire in the place of the patient.

The study was conducted over two periods: at the baseline period the data were obtained when the patients came to the Clinic and consisted of demographic, clinical, and laboratory data and patient's lifestyle; adherence to drug therapy was assessed by the self-report method $(16,17)$, which consisted of the patient's information about which drugs he had taken since the last follow-up visit. After the description given by the patient, the reported drugs were compared to the prescription on the patient's record in order to identify the accuracy of the self-report. When accuracy was not detected, the patient was classified as non-adherent. The patient was also considered to be non-adherent when he reported not taking one dose of the medication, when he spent a day or more without taking the drug, or if he interrupted treatment.

The drugs evaluated were antihypertensive drugs such as beta-blockers, angiotensin-converting enzyme inhibitors,

Table 1. General patient characteristics and adherence at baseline.

\begin{tabular}{|c|c|c|c|c|c|c|}
\hline & General & Adherent & Non-adherent & OR & $95 \% \mathrm{Cl}$ & $\mathrm{P}$ \\
\hline $\mathrm{N}$ & 149 & $82.6 \%(123)$ & $17.4 \%(26)$ & & & \\
\hline Male $[\%(\mathrm{~N})]$ & $60.4 \%(90)$ & $82.2 \%(74)$ & $17.8 \%(16)$ & 0.944 & [0.396-2.250] & $0.54^{*}$ \\
\hline Age (years; mean \pm SD) & $51 \pm 16.7$ & $49 \pm 16.8$ & $59 \pm 14.1$ & - & - & $0.006^{* *}$ \\
\hline Marital status - married ${ }^{+}$ & $61.1 \%(91)$ & $80.2 \%(73)$ & $19.8 \%(18)$ & 0.649 & {$[0.262-1.608]$} & $0.238^{*}$ \\
\hline \multicolumn{7}{|l|}{ Education } \\
\hline Up to primary education & $67.1 \%(100)$ & $82.0 \%(82)$ & $18.0 \%(18)$ & 1.125 & {$[0.451-2.804]$} & $0.498^{*}$ \\
\hline High school and college & $32.9 \%(49)$ & $83.7 \%(41)$ & $16.3 \%(8)$ & & & \\
\hline \multicolumn{7}{|l|}{ Personal income } \\
\hline Up to a minimum wage & $62.4 \%(93)$ & $81.7 \%(76)$ & $18.3 \%(17)$ & 1.168 & [0.482-2.833] & $0.457^{*}$ \\
\hline$>$ a minimum wage & $37.6 \%(56)$ & $83.9 \%(47)$ & $16.1 \%(9)$ & & & \\
\hline Retired $\ddagger$ & $55.7 \%(83)$ & $80.7 \%(67)$ & $19.3 \%(16)$ & 0.748 & {$[0.314-1.778]$} & $0.331^{*}$ \\
\hline Pills/day (N) & $6.7 \pm 4.0$ & $6.3 \pm 3.9$ & $8.5 \pm 4.3$ & - & - & $0.008^{\star *}$ \\
\hline Smoking & $10.8 \%(16)$ & $75.0 \%(12)$ & $25.0 \%(4)$ & 0.600 & {$[0.177-2.034]$} & $0.300^{*}$ \\
\hline Serum creatinine (mean \pm SD) & $1.93 \pm 1.0$ & $1.84 \pm 0.91$ & $2.32 \pm 1.25$ & - & - & $0.030^{\star *}$ \\
\hline Estimated GFR $\left(\mathrm{mL} \cdot \mathrm{min}^{-1} \cdot 1.73\left(\mathrm{~m}^{2}\right)^{-1}\right)$ & $48.9 \pm 28.4$ & $52.10 \pm 29.31$ & $35.24 \pm 19.03$ & - & - & $0.01^{* \star}$ \\
\hline Heart disease & $10.7 \%(16)$ & $62.5 \%(10)$ & $37.5 \%(6)$ & 0.295 & {$[0.096-0.902]$} & $0.037^{*}$ \\
\hline Drug self-administration & $77.9 \%(116)$ & $87.9 \%(102)$ & $12.1 \%(14)$ & 4.163 & {$[1.688-10.269]$} & $0.002^{*}$ \\
\hline
\end{tabular}

${ }^{+}$Single, widowed, and divorced patients with a stable consensual union were also considered to be married. ‡All patients on sick pay or retirement (by age or disability) were considered to be retired. Glomerular filtration rate was estimated using the Modification of Diet in Renal Disease formula (GFR $\left(\mathrm{mL} \cdot \mathrm{min}^{-1} 1.1 .73\left(\mathrm{~m}^{2}\right)^{-1}\right)=186 \times(\mathrm{Pcr})^{-1.154} \times(\text { age })^{-0.203} \times(0.742$ if female) $\times\left(1.210\right.$ if African-American). ${ }^{*}$ Fisher exact test; ${ }^{*}$ Student $t$-test. The percent of adherent and non-adherent patients refer to absolute number for each variable considering all patients. 
angiotensin-receptor blockers, calcium channel blockers, and diuretics, in addition to statins and acetylsalicylic acid. Patients who reported the drugs and number of units in use consistent with the prescription made in the visit that preceded the interview, were considered to be adherent. A reassessment of adherence to drug therapy was done 1 year later via telephone interview.

At the variable adherence crossover, a dichotomous categorical variable with metric, continuous and normally distributed variables, we applied the Student $t$-test; with polychotomous categorical variables, we used the $x^{2}$ test, and with dichotomous variables, we used the Fisher exact test. We used logistic regression, adjusted for statistically significant variables in univariate analysis and, to compare adherence throughout the studied stages, we used the McNemar test $(18,19)$. In univariate analysis, $P<0.05$ was considered to be significant.

\section{Results}

Table 1 presents the data for 149 patients assessed at baseline. There was a prevalence of males $(60.4 \%)$, mean age was $51 \pm 16.7$ years, the majority was married $(61.1 \%)$ and had a mean educational up to primary education $(67.1 \%)$. Most had personal incomes below the poverty level (62.4\%), and $55.7 \%$ were retired. The mean glomerular filtration rate (GFR), estimated by the Modification of Diet in Renal Disease formula for the group, was $48.9 \pm 28.4$ $\mathrm{mL} \cdot \mathrm{min}^{-1} \cdot 1.73\left(\mathrm{~m}^{2}\right)^{-1}$. Most patients self-administered their own medication $(77.9 \%)$ and the average daily number of tablets per patient was $6.7 \pm 4.0$.

Non-adherent patients had a higher mean age, were using a larger amount of pills per day at baseline and at the final period, did not self-administer medications, had higher mean serum creatinine, lower GFR, and a lower frequency of coronary heart disease. Table 1 shows that among 116 $(77.9 \%)$ patients who self-administered their medication, $14(12.1 \%)$ were non-adherent and 102 (87.9\%) were adherent. In $16(10.7 \%)$ of the 149 patients who presented coronary disease, $6(37.5 \%)$ were non-adherent and 10 $(62.5 \%)$ were adherent.

Compared to baseline, the percentage of non-adherence increased from 17 to $27 \%(P=0.04)$ among patients initially adherent, and, more importantly, decreased in $50 \%$ in patients who were initially non-adherent (Figure 1).

The logistic regression model, adjusted for statistically significant variables in univariate analysis, showed that intake of five or more tablets per day, as well as drug administration by caregivers, were significantly associated with patient's non-adherence (Table 2).

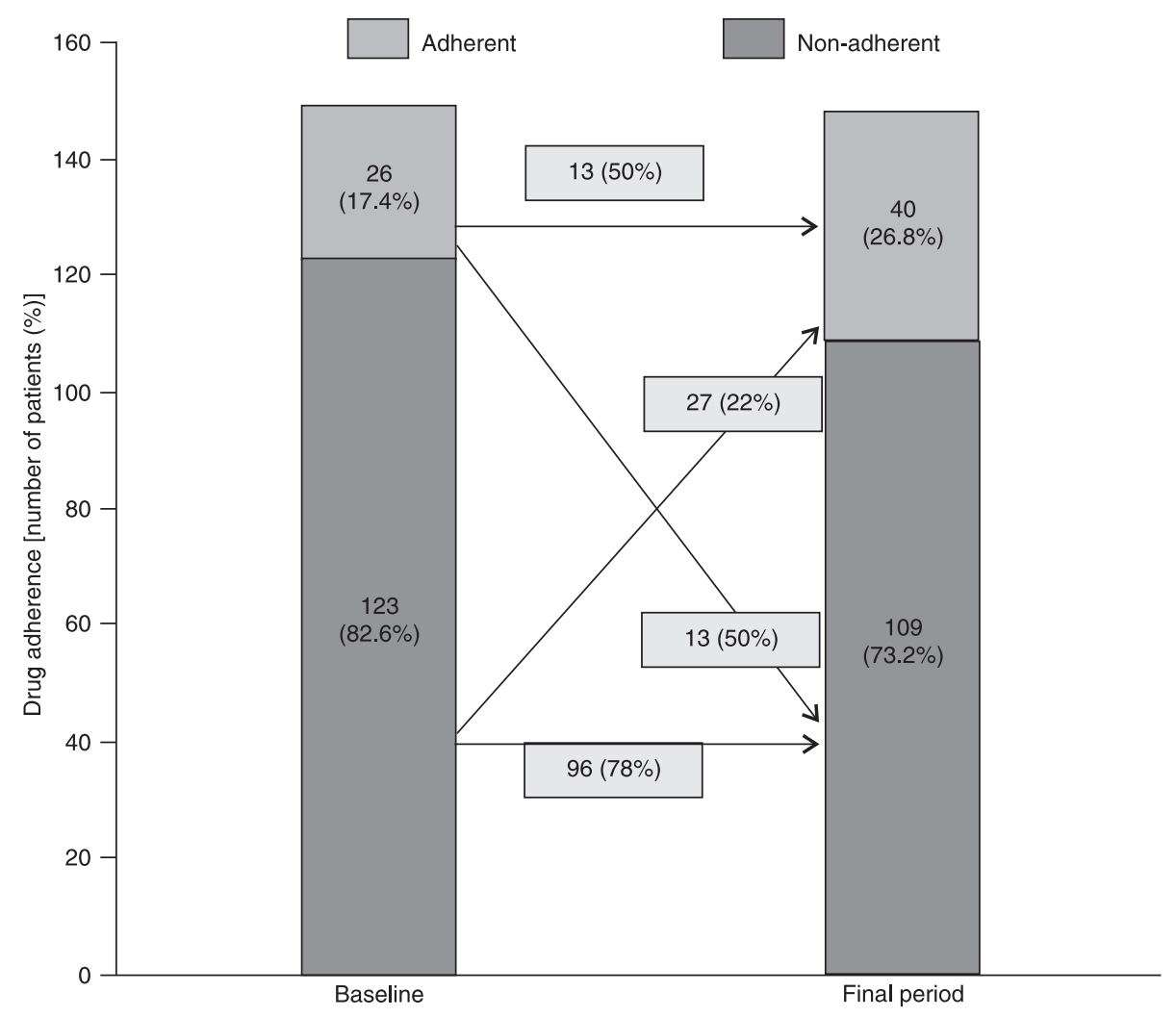

Figure 1. Drug adherence at the beginning (baseline) and at the end (final period) of the study. 
Table 2. Logistic regression for non-adherence at baseline.

\begin{tabular}{lccc}
\hline & OR & $0.95 \% \mathrm{Cl}$ & $\mathrm{P}$ \\
\hline Number of daily pills $>5$ & 2.71 & {$[0.99-7.41]$} & 0.052 \\
Drug administration by caregivers & 3.53 & {$[1.39-8.89]$} & 0.007 \\
\hline
\end{tabular}

Data were adjusted for age, number of tablets/day, serum creatinine, estimated glomerular filtration rate, comorbid coronary artery disease, administration of medication by caregivers.

\section{Discussion}

In the present study, non-adherence to drug therapy among patients with kidney disease who were not on dialysis was found to be associated with a larger number of pills taken per day and drug administration by a caregiver.

Non-adherence to medication in chronic illness significantly increases morbidity and mortality, especially in developing countries $(3,4)$. The measurement of non-adherence and the risk factors associated with it aim to develop interventions to improve adherence (5). Non-adherence to drug therapy has multifactorial origins (11).

Most studies on adherence to drug therapy in renal diseases are conducted on post-transplant patients (20) and evaluate the percentage of non-adherence to immunosuppressants and outcomes such as death and graft loss (21). So far, only two studies have been published on the evaluation of adherence by patients with kidney disease not yet on dialysis $(22,23)$, a fact that justifies the relevance of the present study.

There is no satisfactory gold standard method for assessing adherence to drug therapy, since all methods have limitations related to low sensitivity and specificity (5). We used the self-reported method to test patients' adherence, which was applied during a visit to the clinic and by telephone. Since this method is easy to use and interpret, it can be incorporated into the management of patients with kidney disease.

Comparisons of results from this study with previously published reports are difficult due to the great diversity of methods used in assessing adherence (24) and the scarcity of publications on patients with clinical conditions similar to those of our patients. For example, Moreira et al. (22), using the self-report method and a drug profile similar to the one used by us, found a non-adherence rate of $18.5 \%$ in non-dialysis patients. Likewise, Lee et al. (23), based on two methods, pill count and electronic monitoring, observed a non-adherence rate of $18 \%$. These percentages are similar to those found in our study at the baseline period (17.8\%).

Similarly to what has been described in other studies $(13,17)$, we also observed that the number of non-adherent patients increased throughout the study (up to $26.8 \%$ ), but it is worth noting that the percentage of adherent patients who became non-adherent (22\%) was lower than the per- centage of non-adherent patients who became adherent $(50 \%)$, possibly demonstrating the educational role of the interdisciplinary team in monitoring patients with CKD.

Regarding the demographic variables of our sample, there was a prevalence of low educational level and of low income, but gender, marital status, education, retirement, and personal income, identified as risk of noncompliance in other publications $(17,23)$, were not statistically significant risks in our study.

Another possibility that may explain these findings is that, in standard patient care, the staff seeks to provide guidance using accessible language about the importance of regular schedules, medication storage, and most frequent side effects, besides encouraging the use of mechanisms that help the patient to remember taking the medications, such as cell phone, alarm clocks. Although our study was not designed to evaluate the role of interdisciplinary care in adherence to drug therapy, recent studies have shown that patients treated by a multidisciplinary team show better clinical and laboratory parameters at the beginning of dialysis compared to those who receive medical care only from nephrologists $(24,25)$. These findings are possibly related to greater adherence to the clinical guidelines received.

Impaired renal function observed in progressive kidney disease, particularly in more advanced stages, is associated with complications and comorbidities that require prescription of many different drugs. The impact of the number of drugs and of renal function on adherence to drug therapy has been studied mainly in kidney transplant patients but has been underappreciated in patients with kidney disease not yet on dialysis. Moreira et al. (22) observed that the average number of daily pills ( $\geq 4$ pills per day) and the more advanced stages of chronic kidney disease (stages 4 and 5) were statistically associated with non-adherence to drug therapy. In our study, the association of degree of renal dysfunction and non-adherence to drug therapy was only observed in univariate analysis, whereas the association with number of pills was statistically significant after adjusting for the variables age, drug self-administration, and comorbidity such as coronary artery disease.

Patients with cognitive decline, a variable with negative impact on adherence (17), were assessed for adherence to drug therapy by means of caregivers. Contrary to our expectation, we found a higher frequency of non-adherence among patients who relied on caregivers (a family member or close friend) to take their medicines compared to those who self-administered their medication.

Although in most cases there is no way to reduce the drug arsenal used for the clinical management of patients with CKD, it is possible to reduce the amount and frequency of drugs, giving priority to long-acting ones administered once daily, and thus minimizing non-adherence to prescribed medications.

In order to improve adherence to drug therapy in patients who depend on caregivers to manage their medications, it is 
necessary to use an accessible language to clearly explain to the caregivers the importance of schedule regularity, medication storage, most frequent side effects, and the use of strategies that may help them to remember taking the medications (26) and we believe that pharmacists could play a key role in the multidisciplinary team in developing these strategies.

Although our study has focused on drug adherence, the concept of adherence defined by the WHO also includes items such as diet and changes in life style (5).

This study had limitations: i) at baseline the interview to

\section{References}

1. K/DOQI clinical practice guidelines for chronic kidney disease: evaluation, classification, and stratification. Am J Kidney Dis 2002; 39: S1-S266.

2. Sesso R, Lopes AA, Thomé FS, Bevilacqua JL, Junior JER, Lugon J. Resultado do Censo de Diálise da Sociedade Brasileira de Nefrologia. J Bras Nefrol 2010; 29 (Suppl 4): 197-202.

3. Burkhart PV, Sabate E. Adherence to long-term therapies: evidence for action. J Nurs Scholarsh 2003; 35: 207.

4. De Geest S, Sabate E. Adherence to long-term therapies: evidence for action. Eur J Cardiovasc Nurs 2003; 2: 323.

5. Sabate E. Adherence to long-term therapies: evidence for action. Geneva: World Health Organization; 2003.

6. Haynes R. Determinants of compliance: the disease and the mechanics of treatment. Baltimore: Johns Hopkins University Press; 1979.

7. Cukor D, Rosenthal DS, Jindal RM, Brown CD, Kimmel PL. Depression is an important contributor to low medication adherence in hemodialyzed patients and transplant recipients. Kidney Int 2009; 75: 1223-1229.

8. Hyre AD, Krousel-Wood MA, Muntner P, Kawasaki L, Desalvo KB. Prevalence and predictors of poor antihypertensive medication adherence in an urban health clinic setting. $J$ Clin Hypertens 2007; 9: 179-186.

9. Failde I, Ramos I. Validity and reliability of the SF-36 Health Survey Questionnaire in patients with coronary artery disease. J Clin Epidemiol 2000; 53: 359-365.

10. Ciconelli RM, Ferraz MB, Santos W, Meinão I, Quaresma MR. Tradução para a língua portuguesa e validação do questionário genérico de avaliação de qualidade de vida SF36 (Brasil SF-36). Rev Bras Reumatol 1999; 39: 143-150.

11. Osterberg L, Blaschke T. Adherence to medication. N Engl J Med 2005; 353: 487-497.

12. Leite SN, Vasconcellos MdPC. Adherence to prescribed therapy: points for concepts and presuppositions discussion. Ciên Saúde Coletiva 2003; 8: 775-782.

13. Vrijens B, Vincze G, Kristanto P, Urquhart J, Burnier M. Adherence to prescribed antihypertensive drug treatments: longitudinal study of electronically compiled dosing histories. BMJ 2008; 336: 1114-1117.

14. Dewulf NL. Investigação sobre a adesão ao tratamento medicamentoso em pacientes com doenças inflamatórias evaluate adherence to drug therapy was assessed by the self-report method and at the end of the study the patients were contacted by telephone; ii) we excluded patients who showed cognitive impairment when tested by the MMSE.

The present study showed that non-adherence to drug medication was identified in $17.4 \%$ of patients with kidney disease not on dialysis and increased during the next 12 months of the disease course to $26.8 \%$. Non-adherence to medication is associated with the intake of a larger number of daily pills and with reliance on drug administration by caregivers. intestinais. [Master's thesis]. São Paulo: Universidade de São Paulo; 2005.

15. Delgado BAL, Lima ML. Contributo para a validação concorrente de uma medida de adesão aos tratamentos. Psicol Saúde Doenças 2001; 2: 81-100.

16. Kulkarni SP, Alexander KP, Lytle B, Heiss G, Peterson ED. Long-term adherence with cardiovascular drug regimens. Am Heart J 2006; 151: 185-191.

17. Grymonpre RE, Didur CD, Montgomery PR, Sitar DS. Pill count, self-report, and pharmacy claims data to measure medication adherence in the elderly. Ann Pharmacother 1998; 32: 749-754.

18. Levin J. Estatística aplicada a ciências humanas. $2^{\mathrm{a}}$ edn. São Paulo: Harper \& Row do Brasil; 1983.

19. Vieira S. Bioestatística: tópicos avançados. Rio de Janeiro: Campus; 2003.

20. Vasquez EM, Tanzi M, Benedetti E, Pollak R. Medication noncompliance after kidney transplantation. Am J Health Syst Pharm 2003; 60: 266-269.

21. Dew MA, DiMartini AF, De Vito DA, Myaskovsky L, Steel J, Unruh $\mathrm{M}$, et al. Rates and risk factors for nonadherence to the medical regimen after adult solid organ transplantation. Transplantation 2007; 83: 858-873.

22. Moreira L, Fernandes P, Monte S, Martins A. Adesão ao tratamento farmacológico em pacientes com doença renal crônica. J Bras Nefrol 2008; 30: 113-119.

23. Lee JY, Greene PG, Douglas M, Grim C, Kirk KA, Kusek JW, et al. Appointment attendance, pill counts, and achievement of goal blood pressure in the African American Study of Kidney Disease and Hypertension Pilot Study. Control Clin Trials 1996; 17: 34S-39S.

24. Curtis BM, Ravani P, Malberti F, Kennett F, Taylor PA, Djurdjev $O$, et al. The short- and long-term impact of multidisciplinary clinics in addition to standard nephrology care on patient outcomes. Nephrol Dial Transplant 2005; 20: 147-154.

25. Goldstein M, Yassa T, Dacouris N, McFarlane P. Multidisciplinary predialysis care and morbidity and mortality of patients on dialysis. Am J Kidney Dis 2004; 44: 706-714.

26. Brasil. Ministério da Saúde. Guia prático do cuidador. Brasília: Ministério da Saúde; 2008. 